\title{
ЭПИЛЕПТОЛОГИЯ
}

УДК 616.853-056.3:616.891.6:616.895.3:616.89-008.441.44

Для цитирования: Шова Н.И., Михайлов В.А. Тревожное расстройство как фактор риска суицидальных намерений у пациентов с эпилепсией. Сибирский вестник психиатрии и наркологии. 2019; 4 (105): 89-94. https://doi.org/10.26617/1810-3111-2019-4(105)-89-94

\section{Тревожное расстройство как фактор риска суицидальных намерений у пациентов с эпилепсией}

\author{
Шова Н.И., Михайлов В.А.
}

Наџиональный медицинский исследовательский центр психиатрии и неврологии им. В.М. Бехтерева Россия, 192019, Санкт-Петербург, ул. Бехтерева, 3

\section{PEЗЮME}

Тревожные расстройства широко распространены среди пациентов, страдающих эпилепсией. В современной литературе представлены многочисленные исследования о влиянии эмоциональных и аффективных расстройств на риск формирования суицидальных намерений, но все они сосредоточены преимущественно на депрессивном расстройстве. Материал и методы. Нами обследовано 102 пациента с диагнозом эпилепсии. Общая выборка была разделена на две группы: 1-я группа - пациенты без суицидальных намерений (N=73), 2-я группа - пациенты с суицидальными намерениями ( $\mathrm{N}=29)$. Обследование включало изучение анамнеза, клинический и неврологический осмотры, психометрический метод с применением психометрических шкал и опросников. Для скрининга симптомов тревоги использовались Госпитальная шкала тревоги и депрессии (HADS), симптоматический опросник SCL-90-R. C целью оценки риска возникновения суицидальных намерений использовался Опросник суицидального риска (модификация Т.Н. Разуваевой). Результаты. Пациенты с суицидальными намерениями характеризовались достоверно значимым высоким значением тревоги $(9,17$ балла по шкале HADS), что соответствует субклиническому уровню тревоги. Полученные данные по шкале HADS подтверждались результатами опросника SCL-90-R в виде увеличения значений показателей: тревожность - 1,22 балла, соматизация - 1,30 балла. Наличие тревоги $(\mathrm{r}=0,7 ; \mathrm{p}<0,05)$ достоверно ассоциировалось с фактором риска возникновения суицидальных намерений (временная перспектива). Выявлена обратная корреляционная связь между тревогой и антисуицидальным фактором ( $\mathrm{r}=-0,63 ; \mathrm{p}<0,05)$. Наличие тревоги является характерной чертой у пациентов с эпилепсией. Увеличение уровня тревоги повышает риск возникновения суицидальных намерений и обосновано такими показателями как демонстративность, аффективность и временная перспектива. По мере увеличения тревоги отмечается снижение антисуицидального фактора. Полученные нами данные в целом подтверждают высокую распространенность симптомов тревоги у больных с эпилепсией, их высокую коморбидность, а также их влияние на возникновение суицидальных намерений.

Ключевые слова: эпилепсия, тревога, депрессия, суицидальные намерения, непсихотические психические расстройства, психометрическое обследование, демонстративность, аффективность, временная перспектива, антисуицидальный барьер, антисуицидальный фактор.

\section{ВВЕДЕНИЕ}

Повышенное внимание к психической коморбидности при эпилепсии в последние годы в основном было сосредоточено на депрессии, которая, как было продемонстрировано в американских публикациях, широко распространена и влияет на качество жизни пациентов, контроль приступов и риск возникновения суицидальных намерений $[1,2]$.

Многочисленные исследования показали, что значительная часть взрослых и детей с эпилепсией испытывает тревогу значительно больше, чем в общей популяции и при некоторых других хронических заболеваниях. Так, у взрослых пациентов, страдающих эпилепси- ей, оценка распространенности тревожного расстройства варьирует в границах от $11 \%$ до $50 \%[3,4]$. Результаты двух популяционных исследований, проведенных в США, показали, что у страдающих эпилепсией вероятность постановки диагноза тревожного расстройства была примерно в 2 раза выше, чем у людей без эпилепсии, коэффициент распространенности составил $1,61[5,6]$.

Как и взрослые, дети с эпилепсией также испытывают высокий уровень тревоги. По материалам популяционных исследований выявлено, что у 17\% детей с эпилепсией была диагностирована тревога по сравнению с 3\% детей без эпилепсии $[7,8,9]$. 
При исследовании полиморфизмов генов у пациентов с височной эпилепсией выдвинуто предположение о связи между генетическими и нейробиологическими гипотезами эпилепсии и тревоги. Генотипирование полиморфизмов гена транспортера серотонина, выполненное у 155 пациентов с височной эпилепсией, обнаружило, что наличие специфического аллеля $(\mathrm{C}$ аллель 5-HT1А С-1019G полиморфизма) является независимым фактором риска для возникновения тревожного расстройства $[10,11]$. Отмечается связь дисбаланса серотонина с эпилепсией и тревожным расстройством. Результаты позитронно-эмиссионной томографии у пациентов с тяжелой мезиальной височной эпилепсией показали снижение потенциала связывания рецептора серотонина типа $1 \mathrm{~A}$ в очаге эпилептической активности [12].

Тревога оказывает большое влияние на качество жизни больных эпилепсией. Авторами из Великобритании обнаружено, что психиатрическая коморбидность является более значимым фактором, влияющим на качество жизни пациентов с эпилепсией, чем контроль над приступами или тяжесть течения заболевания [13].

В дополнение к влиянию на качество жизни недавно опубликованные данные свидетельствуют о том, что тревога при эпилепсии связана с повышенными экономическими затратами для здравоохранения и общества в целом. Отмечается, что пациенты с фокальной формой эпилепсии и симптомами тревоги с более высокой частотой в течение года посещали врачей амбулаторно, чем пациенты без тревоги или депрессии, а пациенты с тревогой и депрессией с большей долей вероятности были госпитализированы в стационар [14]. Помимо негативных последствий тревоги при эпилепсии для качества жизни пациента, неблагоприятных эффектов приема антиэпилептических препаратов, снижения когнитивных функций, тревога так же связана с повышенным риском смертности от самоубийства [15].

\section{ЦЕЛЬ ИССЛЕДОВАНИЯ}

Выявление распространенности тревожной симптоматики у взрослых пациентов с диагностированной эпилепсией, а также оценка связи тревоги и риска формирования суицидальных намерений у таких пациентов.

\section{МАТЕРИАЛЫ И МЕТОДЫ}

В общую выборку исследования было включено 102 пациента с установленным диагнозом эпилепсии. В зависимости от наличия пассивных суицидальных намерений пациенты разделены на две группы. В 1-ю группу - контрольную $(\mathrm{N}=73)$ вошли пациенты без суицидальных намерений (48 женщин и 25 мужчин). Ко 2-й группе - исследовательской $(\mathrm{N}=29)$ были отнесены пациенты с пассивными суицидальными намерениями на момент проведения исследования (5 женщин и 24 мужчины).

Исследование проводилось с применением стандартного неврологического осмотра, стандартизованного психиатрического интервью с применением шкал и опросников.

Отдельные показатели клинико-социальной характеристики пациентов 1-й и 2-й групп исследования представлены в таблице 1.

Т а б л и ц а 1 . Клинико-социальная характеристика пациентов 1-й (контрольной) и 2-й (исследовательской) групI

\begin{tabular}{|c|c|c|c|}
\hline \multicolumn{2}{|c|}{ Показатель } & 1-я группа $(\mathrm{M} \pm \mathrm{m})$ & 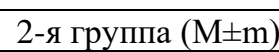 \\
\hline \multicolumn{2}{|c|}{ Средний возраст обследованных лиц, лет } & $35 \pm 4,8$ & $28 \pm 2,8$ \\
\hline \multirow{2}{*}{ Гендерная принадлежность } & Женщины (N) & 48 & 5 \\
\hline & Мужчины (N) & 25 & 24 \\
\hline \multicolumn{2}{|c|}{ Средняя длительность заболевания, лет } & $7 \pm 2,3$ & $14 \pm 5,5$ \\
\hline \multicolumn{2}{|c|}{ Средний возраст начала эпилепсии, лет } & $20 \pm 6,1$ & $10 \pm 1,8$ \\
\hline \multicolumn{4}{|c|}{ Характеристика течения основного заболевания } \\
\hline \multicolumn{4}{|c|}{\begin{tabular}{l|l} 
Частота эпилептических приступов: & \\
\end{tabular}} \\
\hline \multirow{2}{*}{\multicolumn{2}{|c|}{$1-2$ раза в год $(\mathrm{N})$}} & 41 & - \\
\hline & & 25 & 7 \\
\hline \multicolumn{2}{|l|}{$\begin{array}{l}\text { 1-3 раза в месяц (N) } \\
\text { еженедельные }(\mathrm{N})\end{array}$} & 7 & 11 \\
\hline \multicolumn{2}{|l|}{ ежедневные (N) } & - & 9 \\
\hline \multicolumn{2}{|l|}{ несколько раз в сутки (N) } & - & 2 \\
\hline \multicolumn{4}{|c|}{ Характер эпилептических приступов: } \\
\hline \multicolumn{2}{|c|}{ Фокальные приступы без потери сознания } & 56 & 1 \\
\hline \multicolumn{2}{|c|}{ Фокальные приступы с потерей сознания } & 17 & 20 \\
\hline \multicolumn{2}{|c|}{ Генерализованные моторные приступы } & 6 & 7 \\
\hline \multicolumn{2}{|c|}{ Генерализованные не моторные приступы } & 0 & 1 \\
\hline
\end{tabular}


В работе использованы следующие психометрические методики: Опросник психопатологической симптоматики (SCL-90-R), Госпитальная шкала тревоги и депрессии (HADS), Опросник суицидального риска в модификации Т.Н. Разуваевой.

Результаты исследования обрабатывались с использованием программной системы Statistica for Windows (версия 8.0). Данные были представлены в виде средних арифметических со стандартными квадратичными отклонениями $(\mathrm{M} \pm \mathrm{m})$. Средние групповые значения, стандартное отклонение были рассчитаны с помощью модуля описательной статистики. Достоверность различий определялась по критерию Стьюдента $\mathrm{t}$, при котором значения $\mathrm{p} \leq 0,05$ считались достоверными. Взаимосвязь двух показателей осуществлялась по коэффициенту корреляции Пирсона $\mathrm{r}$.

\section{РЕЗУЛЬТАТЫ}

Результаты оценки эмоциональных и аффективных расстройств в соответствии с Госпитальной шкалой тревоги и депрессии (HADS) приведены в таблице 2.

Т а б л и ц а 2. Средние значения показателей тревоги и депрессии по Госпитальной шкале тревоги и депрессии (HADS) у пациентов 1-й (контрольной) и 2-й (исследовательской) групI

\begin{tabular}{|l|c|c|c|}
\hline \multicolumn{1}{|c|}{ Показатель } & 1-я группа & 2-я группа & $\mathrm{p}$ \\
\hline Тревога & 6,00 & 9,17 & $\leq 0,05$ \\
\hline Депрессия & 5,48 & 7,00 & \\
\hline
\end{tabular}

Значения тревоги и депрессии у пациентов 1-й группы (без суицидальных намерений) находятся в пределах референсных значений, соответствующих норме (варьируют от 0 до 7 баллов). Во 2-й группе выявлено достоверно значимое увеличение по показателю тревоги
(9,17 балла) по сравнению с 1-й группой $(6,00$ балла). Полученные результаты подтверждаются по опроснику SCL-90-R. Расширенный спектр выявленных психопатологических проявлений с оценкой тяжести симптомов представлен в таблице 3.

Т а б л и ц а 3. Средние значения показателей психопатологической симптоматики по опроснику SCL-90-R у пациентов 1-й (контрольной) и 2-й (исследовательской) групп

\begin{tabular}{|l|c|c|c|}
\hline \multicolumn{1}{|c|}{ Показатель } & 1-я группа $(\mathrm{M} \pm \mathrm{m})$ & 2 -я группа $(\mathrm{M} \pm \mathrm{m})$ & $\mathrm{p}$ \\
\hline Соматизация & $0,68 \pm 0,27$ & $1,30 \pm 0,46$ & $\leq 0,05$ \\
\hline Обсессивность-компульсивность & $0,85 \pm 0,44$ & $1,44 \pm 0,45$ & \\
\hline Межличностная сенситивность & $0,77 \pm 0,34$ & $1,16 \pm 0,42$ & \\
\hline Депрессия & $0,66 \pm 0,35$ & $1,40 \pm 0,51$ & \\
\hline Тревожность & $0,57 \pm 0,24$ & $1,22 \pm 0,35$ & $\leq 0,05$ \\
\hline Враждебность & $0,54 \pm 0,16$ & $1,05 \pm 0,32$ & \\
\hline Фобичность & $0,41 \pm 0,20$ & $0,81 \pm 0,23$ & \\
\hline Паранойяльность & $0,51 \pm 0,19$ & $0,99 \pm 0,44$ & \\
\hline Психотизм & $0,34 \pm 0,12$ & $0,73 \pm 0,11$ & \\
\hline Общий индекс тяжести симптомов & $0,62 \pm 0,19$ & $1,18 \pm 0,25$ & $\leq 0,05$ \\
\hline
\end{tabular}

Пациенты с эпилепсией обеих групп характеризуются повышенными значениями следующих показателей: соматизация, обсессивность-компульсивность, межличностная сенситивность, депрессивность, фобичность, психотизм. В частности в отношении пациентов 2-й (исследовательской) группы следует отметить значительное увеличение значений по показателям (в баллах): соматизация - 1,30 балла, обсессивность-компульсивность - 1,44 балла, межличностная сенситивность - 1,16 балла, депрессивность - 1,40 балла, тревожность 1,22 балла, фобичность - 0,81 балла, психотизм - 0,73 балла. Дополнительно во 2-й группе наблюдается увеличение по показателям враждебности $(1,05$ балла) и паранойяльности $(0,99$ балла). При межгрупповом сравнении определяется различие на достоверно значимом уровне $(\mathrm{p} \leq 0,05)$ по шкалам соматизации и тревожности. Общий индекс тяжести симптомов характеризуется так же повышенными показателями в обеих группах $(0,62$ и 1,18 балла) с достоверно значимым преобладанием $(\mathrm{p} \leq 0,05)$ среди пациентов с активными суицидальными намерениями.

При оценке степени суицидального риска получены результаты, представленные в таблице 4. Как показано в таблице, пациенты 2-й группы характеризуются достоверно значимым увеличением $(\mathrm{p} \leq 0,05)$ трех показателей: демонстративность (2 балла), аффективность (3,12 балла) и временная перспектива (2,42 балла). 
Наличие тревоги у пациентов 2-й группы на достоверно значимом уровне ассоциировалось с фактором риска возникновения суицидальных намерений (временная перспектива) $\quad(\mathrm{r}=0,7$; $\mathrm{p} \leq 0,05)$. Обратная достоверно значимая корреляционная связь была обнаружена между тревогой и антисуицидальным фактором ( $\mathrm{r}=-0,63$; $\mathrm{p} \leq 0,05)$.

$\mathrm{T}$ a б л и ц а 4. Значения показателей суицидального риска по опроснику суицидального риска в модификации Т.Н. Разуваевой у пациентов 1-й (контрольной) и 2-й (исследовательской) групII

\begin{tabular}{|l|c|c|c|}
\hline \multicolumn{1}{|c|}{ Показатель } & 1-я группа & 2-я группа & $\mathrm{p}$ \\
\hline Демонстративность & 1,17 & 2,00 & $\leq 0,05$ \\
\hline Аффективность & 2,35 & 3,12 & $\leq 0,05$ \\
\hline Уникальность & 0,94 & 2,10 & \\
\hline Несостоятельность & 1,94 & 2,52 & \\
\hline Социальный пессимизм & 3,64 & 3,68 & \\
\hline Слом культурных барьеров & 0,94 & 0,78 & \\
\hline Максимализм & 0,82 & 0,57 & \\
\hline Временная перспектива & 1,17 & 2,42 & $\leq 0,05$ \\
\hline Антисуицидальный фактор & 1,29 & 1,15 & $\leq 0,05$ \\
\hline
\end{tabular}

\section{ОБСУЖДЕНИЕ}

Пациенты с суицидальными намерениями характеризуются ранним началом основного заболевания и продолжительной длительностью эпилепсии. Частота приступов на момент обследования варьировала у них от еженедельных до ежедневных, по семиотике преимущественно имели место фокальные приступы с нарушением осознанности и генерализованные моторные приступы. Данные клинические характеристики указывают на тяжелое течение основного заболевания.

Полученные данные могут отображать «психическую напряженность» и фрустрацию среди пациентов с эпилепсией и суицидальными намерениями, развивающиеся на фоне переживаемого заболевания. Впоследствии с возможным формированием дистресс-синдрома, характеризующегося истощением психических и физических ресурсов организма, дезорганизацией деятельности и поведения.

Обсуждаемые результаты указывают на доминирование эмоционального компонента психопатологического статуса в рамках тревожного расстройства и основного заболевания, а также на готовность пациентов к дезадаптивным формам поведения на фоне невозможности конструктивного планирования своего будущего и сильной погруженности в актуальные проблемы. Они могут быть связаны как с тяжестью течения основного заболевания, так и возможными социальными ограничениями в связи с прессингом стигматизации. Установлено, что повышение степени выраженности тревожных проявлений приводит к снижению витальных мотивов.

\section{ЗАКЛЮЧЕНИЕ}

Таким образом, пациенты с эпилепсией характеризуются наличием тревожного расстройства, которое способно потенцировать возникновение пассивных суицидальных намерений. Данные результаты могут активно использоваться в практической деятельности врачаминеврологами и врачами-психиатрами с целью выделения групп риска пациентов с эпилепсией и суицидальными намерениями и оптимизировать процесс лечения.

\section{КОНФЛИКТ ИНТЕРЕСОВ}

Авторы заявляют об отсутствии явных и потенциальных конфликтов интересов в связи с публикацией данной статьи.

\section{ИСТОЧНИК ФИНАНСИРОВАНИЯ}

Авторы заявляют об отсутствии финансирования при проведении исследования.

\section{СООТВЕТСТВИЕ ПРИНЦИПАМ ЭТИКИ}

Работа соответствует нормам современных этических стандартов, разработанных в соответствии с Хельсинской декларацией Всемирной медицинской ассоциации (протокол заседания локального этического комитета при НМИЦ ПН им. В.М. Бехтерева № ЭК-И-3/19 от 28.01.2019).

\section{ЛИTEPATУPA/REFERENCES}

1. Kanner A.M. Psychiatric issues in epilepsy: the complex relation of mood, anxiety disorders, and epilepsy. Epilepsy Behav. 2009 May; 15:83-7. doi: 10.1016/j.yebeh.2009.02.034

2. Kanner A.M. Anxiety disorders in epilepsy: the forgotten psychiatric comorbidity. Epilepsy Curr. 2011; 11: 90-1. doi: 10.5698/1535-7511-11.3.90

3. Beyenburg S., Mitchell A.J., Schmidt D., Elger C.E., Reuber M. Anxiety in patients with epilepsy: systematic review and suggestions for clinical management. Epilepsy Behav. 2005; 7: 161-71. doi: 10.1016/j.yebeh.2005.05.014 
4. Rai D., Kerr M.P., McManus S., Jordanova V., Lewis G., Brugha T.S. Epilepsy and psychiatric comorbidity: a nationally representative populationbased study. Epilepsia. 2012; 5(3): 1095-103. doi: 10.1111/j.1528-1167.2012.03500.x

5. Kobau R., Gilliam F., Thurman D.J. Prevalence of self-reported epilepsy or seizure disorder and its associations with self-reported depression and anxiety: results from the 2004. Health Styles Survey. Epilepsia. 2006; 47: 1915-21. doi: 10.1111/j.1528-1167.2006.00612.x

6. Ottman R., Lipton R.B., Ettinger A.B., Cramer J.A., Reed M.L., Morrison A. Comorbidities of epilepsy: results from the Epilepsy Comorbidities and Health (EPIC) survey. Epilepsia. 2011; 52:308-15. doi: 10.1111/j.1528-1167.2010.02927.x

7. Russ S.A., Larson K., Halfon N. A national profile of childhood epilepsy and seizure disorder. Pediatrics. 2012; 129: 256-64. doi: 10.1542/peds.20101371

8. Jones J.E., Watson R., Sheth R., Caplan R., Koehn M., Seidenberg M. Psychiatric comorbidity in children with new onset epilepsy. Dev Med Child Neurol. 2007; 49: 493-7. doi: 10.1111/j.14698749.2007.00493.x

9. Hesdorffer D.C., Caplan R., Berg A.T. Familial clustering of epilepsy and behavioral disorders: evidence for a shared genetic basis. Epilepsia. 2012; 53: 301-7. doi: 10.1111/j.1528-1167.2011.03351.x

10. Schenkel L.C., Bragatti J.A., Becker J.A., Torres C.M., Martin K.C., de Souza A.C. Serotonin gene polymorphisms and psychiatry comorbidities in temporal lobe epilepsy. Epilepsy Res. 2012; 99 : 260-6. doi: 10.1016/j.eplepsyres.2011.12.005

11. Kanner A.M. Psychiatric comorbidities and epilepsy: is it the old story of the chicken and the egg? Ann Neurol. 2012; 72: 153-5. doi: 10.1002/ana.23679

12. Savic I., Lindstrom P., Gulyas B., Halldin C., Andree B., Farde L. Limbic reductions of 5-HT1A receptor binding in human temporal lobe epilepsy. Neurology. 2004 Apr 27; 62 (8): 1343-51. DOI: 10.1212/01.wnl.0000123696.98166.af

13. Taylor R.S., Sander J.W., Taylor R.J., Baker G.A. Predictors of health-related quality of life and costs in adults with epilepsy: a systematic review. Epilepsia. 2011; 52: 2168-80. doi: 10.1111/j.15281167.2011.03213.x

14. Hamilton K.T., Anderson C.T., Dahodwala N., Lawler K., Hesdorffer D., French J. Utilization of care among drug resistant epilepsy patients with symptoms of anxiety and depression. Seizure. 2014 Mar; 23(3): 196-200. doi: 10.1016/j.seizure.2013.11.012

15. Gandy M., Sharpe L., Perry K.N., Miller L., Thayer Z., Boserio J. Rates of DSM-IV mood. anxiety disorders. and suicidality in Australian adult epilepsy outpatients: a comparison of well-controlled vs refractory epilepsy. Epilepsy Behav. 2013 Jan; 26(1): 29-35. doi: 10.1016/j.yebeh.2012.10.023

Поступила в редакцию 21.08.2019 Утверждена к печати 02.12.2019

Шова Наталья Игоревна, аспирант, ФГБУ «НМИЦ ПН им. В.М. Бехтерева», ORCID ID 0000-0003-3635-5850.

Михайлов Владимир Алексеевич, доктор медицинских наук, заместитель директора по инновационному научному развитию и международному сотрудничеству, главный научный сотрудник и научный руководитель шестого и седьмого отделений, ФГБУ «НМИЦ ПН им. В.M. Бехтерева». ORCID ID 0000-0002-7700-2704.

Шова Наталья Игоревна, skins_cassi@ outlook.com

Михайлов Владимир Алексеевич, vladmikh@yandex.ru

УДК 616.853-056.3:616.891.6:616.895.3:616.89-008.441.44

For citation: Shova N.I., Mikhailov V.A. Anxiety disorder as a risk factor for suicidal ideation in patients with epilepsy. Siberian Herald of Psychiatry and Addiction Psychiatry. 2019; 4 (105): 89-94. https://doi.org/10.26617/18103111-2019-4(105)-89-94

\section{Anxiety disorder as a risk factor for suicidal ideation in patients with epilepsy}

\section{Shova N.I., Mikhailov V.A.}

Bekhterev National Medical Research Center of Psychiatry and Neurology

Bekhterev Street 3, 192019, St. Petersburg, Russian Federation 


\section{ABSTRACT}

Anxiety disorders are widespread among patients with epilepsy. Numerous studies on the effect of emotional and affective disorders on the risk of the formation of suicidal ideation are presented in the modern literature, but all of them focus mainly on depressive disorder. Material and Methods. We examined 102 patients with the diagnosis of epilepsy. The total sample was divided into two groups: group 1 - patients without suicidal ideation ( $\mathrm{N}=73$ ), group 2 - patients with suicidal ideation $(\mathrm{N}=29)$. The examination included anamnesis, clinical and neurological examinations, psychometric method using psychometric scales and questionnaires. The following symptoms were used to screen for anxiety symptoms: hospital anxiety and depression scale (HADS), symptomatic questionnaire SCL-90-R. In order to assess the risk of suicidal ideation, a suicide risk questionnaire was used (modification by T.N. Razuvaeva). Results. Patients with suicidal ideation were characterized by a reliably significant high value of anxiety (9.17 points on the HADS scale), which corresponded to a subclinical level of anxiety. The data obtained on the HADS scale were confirmed by the results of the SCL-90-R questionnaire in the form of an increase in the values of the indicators: anxiety -1.22 points, somatization -1.30 points. The presence of anxiety $(r=0.7 ; \mathrm{p} \leq 0.05)$ was reliably associated with a risk factor for suicidal ideation (time perspective). An inverse correlation was revealed between anxiety and the anti-suicidal factor $(\mathrm{r}=-0.63 ; \mathrm{p} \leq 0.05)$. The presence of anxiety is a typical feature in patients with epilepsy. An increase in anxiety level elevates the risk of suicidal ideation and is justified by such indicators as demonstrativeness, affectivity and time perspective. As anxiety increases, a decrease in anti-suicidal factor is noted. Our findings generally confirm the high prevalence of anxiety symptoms in patients with epilepsy, their high comorbidity, and their effect on the occurrence of suicidal ideation.

Keywords: epilepsy, anxiety, depression, suicidal ideation, nonpsychotic mental disorders, psychometric investigation, demonstrativeness, affectivity, time perspective, anti-suicidal barrier, anti-suicidal factor.

Received August 21.2019

Accepted December 02.2019

Shova Natalya I., postgraduate student, Bekhterev National Medical Research Center of Psychiatry and Neurology, ORCID ID 0000-0003-3635-5850.

Mikhailov Vladimir Alekseevich, MD, Deputy Director for Innovative Scientific Development and International Cooperation, Chief Researcher and Scientific Advisor of the sixth and seventh departments, Bekhterev National Medical Research Center of Psychiatry and Neurology. ORCID ID 0000-0002-7700-2704.

Shova Natalya I., skins_cassi@outlook.com

Mikhailov Vladimir A., vladmikh@yandex.ru 